\title{
Is periprocedural sedation during acute stroke therapy associated with poorer functional outcomes?
}

\author{
C Nichols, ${ }^{1}$ J Carrozzella, ${ }^{2}$ S Yeatts, ${ }^{3}$ T Tomsick, ${ }^{2}$ J Broderick, ${ }^{2}$ P Khatri ${ }^{2}$
}

${ }^{1}$ South Denver Neurosurgery, Littleton Adventist Hospital, Colorado, USA ${ }^{2}$ University of Cincinnati Academic Health Center, Cincinnati, Ohio, USA ${ }^{3}$ Medical University of South Carolina, South Carolina, USA

\section{Correspondence to} Dr C Nichols, South Denver Neurosurgery, Littleton Adventist Hospital, 7780 South Broadway, Suite 260, Littleton, CO 80122, USA;

cwnich@gmail.com

Received 13 October 2009 Accepted 15 October 2009 Published Online First 17 December 2009

\begin{abstract}
Background To safely perform acute intra-arterial revascularization procedures, use of sedative medications and paralytics is often necessary. During the conduct of the Interventional Management of Stroke trials (I and II), the level of sedation used periprocedurally varied. At some institutions, patients were paralyzed and intubated as part of the procedural standard of care while at other institutions no routine sedation protocol was followed. The aim of this study was to identify patient characteristics that would correlate with the need for deeper sedation and to explore whether levels of sedation relate to patient outcome.

Methods 75 of 81 patients in the Interventional Management of Stroke II Study were studied. Patients had anterior circulation strokes and underwent angiography and/or intervention. Four sedation categories were defined and tested for factors potentially associated with the level of sedation. Clinical outcomes were also analyzed, including successful angiographic reperfusion and the occurrence of clinical complications. Results Only baseline National Institutes of Health Stroke Scale varied significantly by sedation category $(p=0.01)$. Patients that were in the lower sedation category fared better, having a higher rate of good outcomes $(p<0.01)$, lower death rates $(p=0.02)$ and higher successful angiographic reperfusion rates $(p=0.01)$. There was a significantly higher infection rate in patients receiving heavy sedation or pharmacologic paralysis $(p=0.02)$ and a trend towards fewer groin related complications.
\end{abstract}

Conclusion In this small sample, patients not receiving sedation fared better, had higher rates of successful angiographic reperfusion and had fewer complications. Further examination of the indications for procedural sedation or paralysis and their effect on outcome is warranted.

\section{BACKGROUND}

A myriad of clinical factors govern outcomes in patients with stroke. While initial stroke severity, age, timeliness of thrombolytic therapy and reperfusion are well established predictors, others are poorly understood or not yet identified. ${ }^{1-3}$ As intraarterial procedures become used more frequently in clinical practice to treat acute ischemic stroke, defining procedure related variables that affect patient outcome is paramount.

During the conduct of the Interventional Management of Stroke (IMS) pilot trials (I and II), we noted that the level of sedation used periprocedurally varied greatly. ${ }^{4}$ At some institutions, patients were electively paralyzed and intubated as part of the procedural standard of care while at other institutions no routine sedation protocol was followed.

To safely perform acute intra-arterial revascularization procedures, use of sedative medications and paralytics is often necessary. Unwanted patient movement during catheter manipulation could have disastrous consequences. On the other hand, stroke recovery may be adversely affected by sedatives, paralytics and intubation, both directly, due to direct CNS effects, or indirectly, by leading to medical complications such as ventilator related pneumonia. Delays in the start of the intra-arterial procedure can also occur while waiting for anesthesia to provide deep sedation and intubation. Unfortunately, teasing out the effect of individual drugs, practices of medication administration and decisions regarding intubation is difficult. Furthermore, dosing, duration and timing may be important with regard to the occurrence of adverse effects. ${ }^{6} 7$

We retrospectively analyzed the IMS II trial to: (1) document the variability in sedation patterns used for intra-arterial revascularization procedures; (2) identify clinical factors that were associated with the use of higher levels of sedation; and (3) determine whether use of higher sedation was associated with poorer clinical outcome or the occurrence of complications.

\section{MATERIALS AND METHODS}

The IMS II trial was a 13 center, open label, single arm pilot study of moderate to severe ischemic strokes (National Institutes of Health Stroke Scale $\geq 10$ ) treated with combined intravenous/intraarterial recombinant tissue plasminogen activator (rt-PA) therapy within $3 \mathrm{~h}$ of stroke symptom onset. Details of the trial design and results have been published previously. ${ }^{4}$ In brief, the objectives of the trial were to: (1) obtain reliable estimates of the safety of combing low dose intravenous rt-PA $(0.6 \mathrm{mg} / \mathrm{kg})$ followed by delivery of additional intraarterial rt-PA (up to $22 \mathrm{mg}$ ) and low energy ultrasound via the EKOS microcatheter; (2) consider the efficacy of combined intravenous/intra-arterial rt-PA treatment at 3 months compared with the 3 month outcomes of placebo treated patients in the National Institute of Neurological Diseases and Stroke rt-PA trial; and (3) determine whether the recanalization rate of combined intravenous rt-PA followed by intra-arterial rt-PA and low intensity ultrasound energy is greater than the rate of recanalization for the IMS I study subjects treated only with combined intravenous/intra-arterial rt-PA via a standard microcatheter. ${ }^{13}$

Subjects were treated with low dose intravenous rt-PA and concurrently taken to angiography for 
potential intra-arterial rt-PA therapy. If no thrombus was seen on angiography, no further treatment was provided. As part of the study protocol, clinicians performed formal National Institutes of Health Stroke Scale (NIHSS) examinations just prior to intravenous treatment, again just before initiation of intraarterial therapy and then at completion of intra-arterial therapy. As part of the NIHSS examination, clinicians were asked to determine: (1) whether patients were sedated; (2) whether sedation affected scoring on the NIHSS; and (3) whether the patient was intubated and/or paralyzed. For the purposes of this analysis, we defined four levels of sedation: (1) 'no sedation', cases where no sedative medications were received; (2) 'mild sedation', cases where sedative medications were administered but sedation did not affect the patient's examination by clinical judgment; (3) 'heavy sedation', cases where sedation did affect the examination by clinical judgment; and (4) 'pharmacological paralysis', cases where patients were intubated and/or paralyzed. We also considered sedation as a dichotomous variable consisting of 'low sedation' if in the first two categories and 'heavy sedation' if in the latter two categories. Sedation categories were determined based on the NIHSS evaluation immediately after the angiographic procedure. In five of 75 cases, no sedation score was available post-angiography, and the pre-angiography sedation score was used instead.

We limited the analysis to all IMS II cases with anterior circulation strokes that underwent angiography and/or intervention. Our primary interest was to determine baseline characteristics associated with sedation level. Because of small cell sizes, Fisher's exact tests were used to test for associations between categorical variables. Analysis of variance models were used to test for associations between categorical and continuous variables; to account for small cell sizes, or where the residuals appeared to violate the normality assumption of the analysis of variance model, the non-parametric Kruskal-Wallis test was used instead.

As a secondary analysis, we used logistic regression to determine whether sedation (no/mild sedation versus heavy sedation/ pharmacological paralysis) was associated with good outcome (modified Rankin Score 0-2), death or successful angiographic reperfusion (Thrombolysis in Myocardial Infarction (TIMI) Grade 2-3). Baseline characteristics associated with outcome were considered for inclusion in the multivariable model, and a stepwise selection procedure was used to select covariates for inclusion in the final model.

We also analyzed complications that occurred with respect to sedation category. Rates of symptomatic and asymptomatic intracerebral hemorrhage, significant infections (including pneumonia, sepsis and other infections, but excluding urinary tract infections), access site related complications (including groin hematomas, local bleeding, arterial occlusions and retroperitoneal hematomas), cervical or intracranial vessel dissection or perforation, and acute myocardial infarction were compared.

\section{RESULTS}

Of the 81 patients in the IMS II study, 78 met the inclusion criteria for this analysis. Three of these 78 cases were excluded because sedation data were not available before or after angiography. Among the 75 remaining cases, 40 (53\%) received no sedation and 17 (23\%) were pharmacologically paralyzed. Baseline characteristics across the four sedation categories are presented in table 1 . Only baseline NIHSS varied significantly between the different levels of sedation ( $p=0.03)$. Using dichotomized sedation categories, there was a trend towards higher sedation categories being associated with aphasia, internal carotid artery occlusion and longer procedure duration $(p \leq 0.06)$. Because of the small numbers of patients per center, no analysis of center specific sedation patterns was performed.

Table 2 lists outcomes as defined by sedation category. Patients that were in the lower sedation category fared better, having a higher rate of good outcomes, a lower death rate and more frequent successful reperfusion.

\section{Good outcome (modified Rankin Score 0-2)}

Sedation $(p<0.01)$, gender $(p<0.01)$ and baseline NIHSS $(\mathrm{p}<0.01)$ were associated with outcome in univariate analyses. Only mild or no sedation (OR 5.7; 95\% CI 1.8 to 17.8 ; $\mathrm{p}<0.01$ ) and male gender (OR 4.2; 95\% CI 1.5 to 12.3 ; $p<0.01$ ) were independently associated with good clinical outcome.

\section{Death}

Sedation $(p=0.02)$, baseline NIHSS $(p=0.03)$, baseline systolic blood pressure $(p=0.05)$ and baseline glucose $(p=0.05)$ were associated with death in univariate analyses. The sedation category of heavy sedation or pharmacological paralysis (OR 5.0; 95\% CI 1.3 to $18.7 ; p=0.02$ ) was the only independent predictor of death.

\section{Angiographic reperfusion}

Of the 75 patients included in our analysis, 22 additional patients were excluded from the analysis of reperfusion because they did not undergo revascularization therapy and therefore had no TIMI grades. Sedation $(\mathrm{p}=0.01)$, baseline NIHSS $(p=0.03)$ and internal carotid artery occlusion $(p=0.04)$ were associated with reperfusion in univariate analyses. Mild or no sedation (OR 3.9; 95\% CI 1.1 to $13.9 ; p=0.04$ ) and no ICA occlusion (OR 6.1; 95\% CI 1.3 to $27.9 ; \mathrm{p}=0.02$ ) were the only independent predictors of successful reperfusion.

\section{Complications}

Post-stroke and post-procedural complications were compared between dichotomized sedation groups as above, and are presented in table 3 . We found a significantly higher rate of pneumonia and/or sepsis in patients receiving heavy sedation or pharmacologic paralysis $(p=0.02)$. There were highly nonsignificant trends towards more symptomatic and asymptomatic hemorrhages and fewer access site related complications in the groups receiving heavy sedation or paralysis.

\section{DISCUSSION}

To our knowledge, our report is the first formal examination of the issue of procedural sedation and intubation within a controlled acute interventional ischemic stroke trial. Our data add to the considerable discussion in the literature regarding the potential beneficial and untoward effects of sedative medications on patient outcome and stroke recovery. In particular, the intensive care and anesthesia literatures have shown that continuous use of sedative medications prolongs intubation time and lengthens ICU stay. ${ }^{8} 9$ Continuous sedation has also been associated with an increased likelihood of developing pneumonia in intubated patients. ${ }^{10}$ Conversely, the potential neuroprotective benefits of some sedative medications have been investigated for years although they have never been proven to be of benefit in acute stroke. ${ }^{11-13}$

Our goal was to clarify the factors that were associated with the use of higher levels of sedation in patients undergoing angiography. Furthermore, we wanted to discern the association, if any, between the use of sedation and clinical outcomes and the 
Table 1 Characteristics of patients in each sedation category*

\begin{tabular}{|c|c|c|c|c|c|c|}
\hline & $\begin{array}{l}\text { No sedation } \\
(\mathrm{n}=40)\end{array}$ & $\begin{array}{l}\text { Mild sedation } \\
(n=9)\end{array}$ & $\begin{array}{l}\text { Heavy sedation } \\
(n=9)\end{array}$ & $\begin{array}{l}\text { Pharmacological } \\
\text { paralysis }(\mathrm{n}=17)\end{array}$ & $\begin{array}{l}\text { All groups } \\
(\mathrm{n}=75)\end{array}$ & p Value $\dagger$ \\
\hline Age & $64.6 \pm 11.4$ & $63.6 \pm 10.3$ & $64.0 \pm 12.2$ & $62.8 \pm 12.6$ & $64.0 \pm 11.5$ & 0.98 \\
\hline Baseline NIHSS & $17.3 \pm 4.5$ & $19.4 \pm 8.3$ & $21.0 \pm 5.5$ & $21.2 \pm 4.0$ & $18.9 \pm 5.3$ & 0.03 \\
\hline Non-white & $7(17.5)$ & $2(22.2)$ & $1(11.1)$ & $5(29.4)$ & $15(20.0)$ & 0.68 \\
\hline Atrial fibrillation & $11(27.5)$ & $2(22.2)$ & $2(22.2)$ & $3(17.7)$ & $18(24.3)$ & 0.95 \\
\hline Baseline glucose & $120.1 \pm 42.6$ & $116.1 \pm 19.4$ & $169.0 \pm 104.2$ & $125.2 \pm 38.8$ & $126.6 \pm 52.5$ & 0.68 \\
\hline Aphasia present & $19(47.5)$ & $6(66.7)$ & $8(88.9)$ & $11(64.7)$ & $44(58.7)$ & 0.11 \\
\hline ICA occlusion & $7(18.4)$ & $1(11.1)$ & $2(22.2)$ & $8(47.1)$ & $18(24.7)$ & 0.12 \\
\hline Onset to intravenous treatment (min) & $141.6 \pm 33.5$ & $127.7 \pm 38.3$ & $139.1 \pm 28.4$ & $139.0 \pm 25.5$ & $139.1 \pm 31.6$ & 0.75 \\
\hline Received intra-arterial treatment & $25(62.5)$ & $8(88.9)$ & $8(88.9)$ & $12(70.6)$ & $53(70.7)$ & 0.31 \\
\hline Onset to intra-arterial treatment ( $\mathrm{min})$ & $231.6 \pm 55.3$ & $234.6 \pm 55.0$ & $238.5 \pm 35.4$ & $236.8 \pm 57.7$ & $234.3 \pm 51.9$ & 0.99 \\
\hline Duration of procedure (min) & $111.2 \pm 59.3$ & $119.9 \pm 65.9$ & $150.9 \pm 44.4$ & $136.8 \pm 65.7$ & $123.1 \pm 60.5$ & 0.26 \\
\hline
\end{tabular}

${ }^{*}$ Categorical variables are described using $\mathrm{n}(\%)$ and continuous variables are presented as mean \pm SD.

$t p$ Values represent variation across all four sedation categories.

ICA, Internal carotid artery; NIHSS, National Institutes of Health Stroke Scale.

ability to obtain successful reperfusion. Our analysis shows that higher baseline NIHSS scores correlated with the use of deeper sedation during or prior to angiography and/or intervention. Heavily sedated patients were also significantly less likely to obtain successful angiographic reperfusion, as measured by posttreatment TIMI scores, a finding that is perhaps related to higher rates of internal carotid artery occlusions and associated with longer procedural durations. From a clinical standpoint, it is not surprising that we found that patients with the lowest levels of sedation fared better.

We are unable to conclude whether sedation is a cause of poor outcome because of increased complications or impaired recovery or whether patients with large strokes, as measured by higher baseline NIHSS scores, are more likely to be sedated. It is clear that patients with higher baseline NIHSS scores have a poorer clinical outcome, higher morbidity and mortality and greater rates of symptomatic intracerebral hemorrhage. However, sedation category remained a predictor of poor outcome and death when baseline NIHSS score was accounted for in multivariable analysis. It remains possible, therefore, that the use of sedation itself is related to clinical outcome, given our findings. Alternatively, it is also possible that the use of heavier sedation is a non-specific marker for a group of unmeasured clinical factors that predict poor outcome.

From a procedural standpoint, there was a non-significantly lower rate of access site related complications associated with heavier sedation. Whether this difference is found to be significant in additional studies remains to be seen. In this analysis, access site related complications were limited to groin hematomas, a single arterial pseudoaneurysm, a moderate severity common iliac occlusion and/or local bleeding. There were no significant retroperitoneal hemorrhages in this cohort. In practice, the vast majority of the above access site related complications can be treated by manual compression or conservative measures. Based on our analysis, we suggest that reducing the rate of access site related complications should not influence neurointerventional specialists to heavily sedate or pharmacologically paralyze their patients alone.

Conversely, heavy sedation or paralysis may be warranted if patient movement is felt to pose an increased risk of vessel perforation or dissection. In this analysis, the only vessel dissection occurred in a cervical carotid artery in a patient in the heavily sedated group. Whether patient agitation or movement may have contributed to dissection is unknown. Because these complications are rare, however, we are unable to conclude whether greater sedation or paralysis is useful in avoiding these complications.

We did find a significantly higher rate of infection in those patients receiving heavy sedation or pharmacological paralysis. Whether short term or long term use of sedative medications is in part responsible for higher infection rates in those patients is unclear based on this analysis. However, given these findings, we cannot overlook the fact that medication effects may play some role in immunosuppression, aspiration risk or prolongation of intubation time.

This analysis is limited by its small sample size. While we were able to demonstrate a significant association between sedation and outcome, the magnitude of that association is unclear, as evidenced by the wide CIs around the ORs. In a larger population, we might be able to conclude whether sedation

Table 2 Outcomes in patients receiving different levels of sedation

\begin{tabular}{|c|c|c|c|c|c|c|}
\hline & $\begin{array}{l}\text { No sedation } \\
(n=40)(n(\%))\end{array}$ & $\begin{array}{l}\text { Mild sedation } \\
(\mathrm{n}=9)(\mathrm{n}(\%))\end{array}$ & $\begin{array}{l}\text { Heavy } \\
\text { sedation } \\
(n=9)(n(\%))\end{array}$ & $\begin{array}{l}\text { Pharmacological } \\
\text { paralysis } \\
(\mathrm{n}=9)(\mathrm{n}(\%))\end{array}$ & $\begin{array}{l}\text { All groups } \\
(n=9)(n(\%))\end{array}$ & p Value* \\
\hline \multirow[t]{2}{*}{ Death } & $3(7.5)$ & $1(11.1)$ & $2(22.2)$ & $6(35.2)$ & $12(16)$ & 0.02 \\
\hline & & $4(8.2)$ & & $8(30.8)$ & & \\
\hline \multirow[t]{2}{*}{ mRS $0-2$} & $24(60)$ & $6(66.6)$ & $2(22.2)$ & $4(23.5)$ & $36(48)$ & $<0.01$ \\
\hline & & $30(61.2)$ & & $6(23.1)$ & & \\
\hline $\begin{array}{l}\text { Successful reperfusion } \\
\text { (TIMI 2-3) }\end{array}$ & 24/33 (72.7) & & $7 / 20(35)$ & & $31 / 53(58.5)$ & 0.01 \\
\hline
\end{tabular}

*p Values represent dichotomized sedation categories.

mRS, modified Rankin Scale; TIMI, Thrombolysis in Myocardial Infarction. 
Table 3 Complications in patients receiving different levels of sedation

\begin{tabular}{llll}
\hline & $\begin{array}{l}\text { None or mild } \\
\text { sedation } \\
(\mathbf{n}=\mathbf{4 9})(\mathbf{n}(\%))\end{array}$ & $\begin{array}{l}\text { Heavy sedation } \\
\text { or paralyzed } \\
(\mathbf{n}=\mathbf{2 6})(\mathbf{n}(\%))\end{array}$ & p Value \\
\hline Intracerebral hemorrhage & & & \\
$\quad$ Symptomatic & $4(8.2)$ & $5(19.2)$ & 0.26 \\
$\quad$ Asymptomatic & $7(14.3)$ & $9(34.6)$ & 0.07 \\
Infections & $4(8.2)$ & $8(30.8)$ & 0.02 \\
Access site related complications & $12(24.5)$ & $4(15.4)$ & 0.55 \\
Vessel dissection or perforation & 0 & $1(3.9)$ & 0.35 \\
Acute myocardial infection & $2(4.1)$ & $1(3.9)$ & 1 \\
\hline
\end{tabular}

alone appears to influence outcome. Our inability to precisely determine the types of medications, their duration of use or the route of administration in each case also limits this analysis. While we did record all medications used as part of the study, the times of administration with respect to the angiographic procedure were not recorded, nor was their duration of use.

\section{CONCLUSION}

In this study, we characterized the wide variability in the use of sedation in patients undergoing intra-arterial therapy. Initial stroke severity, as measured by the NIHSS score, was highly associated with the use of deeper sedation. Furthermore, the use of sedation was a more potent marker for poor outcome and death than the initial NIHSS score. Additional study is needed to determine if higher levels of sedation simply mark patients with a poorer outcome, or if they impair stroke recovery and lead to iatrogenic complications, as represented by the higher infection rates in this analysis.
Funding The IMS II study was funded by the National Institute of Neurological Diseases and Stroke (NDS No NS39160) and EKOS Corporation.

Competing interests JPB has acted as a consultant for Genentech Inc.

Ethics approval This study was conducted with the approval of the University of Cincinnati institutional review board

Provenance and peer review Not commissioned; not externally peer reviewed.

\section{REFERENCES}

1. The National Institute of Neurological Disorders and Stroke rt-PA Stroke Study Group. Tissue plasminogen activator for acute ischemic stroke. N Engl J Med 1995; 333:1581-7.

2. The National Institute of Neurological Disorders and Stroke rt-PA Stroke Study Group. Generalized efficacy of t-PA for acute stroke: subgroup analysis of the NDS t-PA Stroke Trial. Stroke 1997;28:2119-25.

3. Rha JH, Saver JL. The impact of recanalization on ischemic stroke outcome: a meta analysis. Stroke 2007:38:967-73

4. IMS Study Investigators. Combined intravenous and intra-arterial recanalization for acute ischemic stroke: the Interventional Management of Stroke Study. Stroke 2004:35:904-11.

5. The IMS II Trial Investigators. The Interventional Management of Stroke (IMS) II Study. Stroke 2007:38:2127-35.

6. Goldstein LB. The Sygen in Acute Stroke Study Investigators. Common drugs may influence motor recovery after stroke. Neurology 1995;45:865-71.

7. Goldstein LB. Potential effects of common drugs on stroke recovery. Arch Neurol 1998:55:454-6.

8. Kollef MH, Levy NT, Ahrens TS, et al. The use of continuous i.v. sedation is associated with prolongation of mechanical ventilation. Chest 1998:114:541-8.

9. Kress J, Pohlman A, O'Connor $\mathbf{M}$, et al. Daily interruption of sedative infusions in critically ill patients undergoing mechanical ventilation. N Engl J Med 2000;342:1471-7.

10. Rello J, Diaz E, Roque M, et al. Risk factors for developing pneumonia within 48 hours of intubation. Am J Respir Crit Care Med 1999;159:1742-6.

11. Selman WR, Spetzler RF, Roski RA, et al. Barbiturate coma in focal cerebral ischemia Relationship of protection to timing of therapy. J Neurosurg 1982;56:685-90.

12. Shapiro HM. Barbiturates in brain ischaemia. Br J Anaesth 1985;57:82-95.

13. Schwab S, Spranger M, Schwarz S, et al. Barbiturate coma in severe hemispheric stroke: useful or obsolete? Neurology 1997;48:1608-13. 